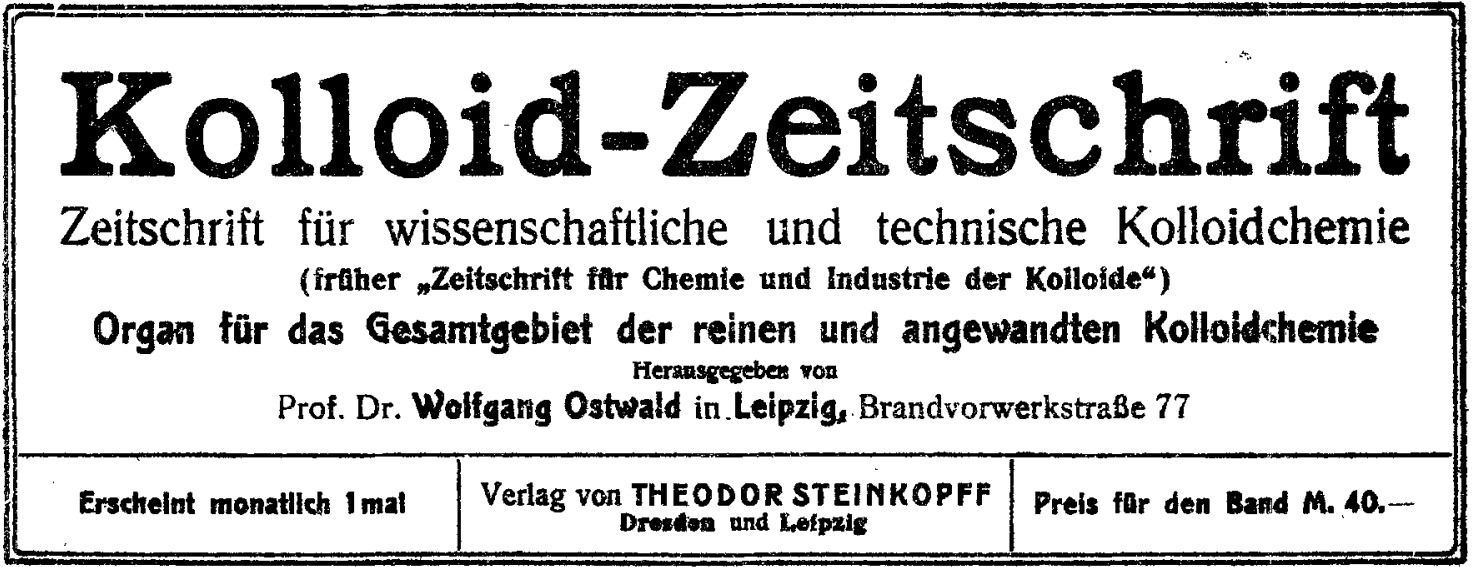

\title{
Uleber den Assoziationsgrad der Molekeln binärer Salze in nichtwässerigen Lösungen. Ein Beitrag zur Kolloidchemie.
}

Von P. Walden (Rostock).

(Eingegangen am 7. Juni 1920.)

Den Ausgangspunkt für die nachstehenden Untersuchungen der Molekulargrōßen gelöster Salze bildeten folgende Annahmen ${ }^{1}$ ): Binäre Salze bilden im geschmoizenen Zustande hochassoziierte Molekeln und weisen Autoionisation auf, in gelöster Form sind sie die besten typischen Elektrolyte. Intramolekulare elektrische Kräfte innerhalb der Einzelmolekel und intermolekulare chemische (elektrische) Kräfte $z w$ is chen den Salzmolekeln an "sich, sowie den Salzmolekeln und den Molekeln des dielektrischen Lösungsmittels sind also die Paktoren, welche die lonenspaltung regeln. Betrachtet man die Salzmolekeln für sich, so ist das wahre, von ihnen ausgefallte Volum $=\frac{M}{d}\left(\frac{\varepsilon-1}{\varepsilon+2}\right)$, wenn $\varepsilon$ die Dielektrizitätskonstante bedeutet und $\frac{M}{d}$ das scheinbare Molekularvolum ist. Nun haben aber Messungen der Dielektrizitätskonstanten gelöster Salze in schlechten Ionisatoren ergeben, daB für diese typischen starken Elektrolyte das wahre Volum gleich ist dem scheinbaren, d. h., daß diese Molekeln den ihnen zur Verfügung gestellten Raum anscheinend ganz ausfüllen, - - dem Maximum der molekularen Raumerfüllung entspricht dann ein Minimum an freier Schwingung und ein Maximum an Spannung in der Molekel. Tritt jetzt ein Dielektrikum als Lösungsmittel hinzu, so wird, je nach dessen Eigenschaften, die Zerteilung oder Zersplitterung der polymeren Salzmolekeln teils zu kleineren Molekeln,

1) P. Wal de n, Bu!1. Acad. Sc. St. Petersb. 1912, 305, 1055; Journ. Ámer. Chem. Soc. 35, 1649 (1913). teils $z \mathrm{u}$ Ionen einsetzen müssen. Je geringer die Dielektrizitätskonstante (Dissoziationskraft) des Lösungsmittels ist, um so weniger wird die Aufspaltungs- und Entspannungstendenz der Salzmolekeln sich betătigen können, um so mehr werden wir dem normalen Assoziationsbzw. Polymeriegrade der Elektrolytmolekeln uns nähern. Aendern wir das Medium und steigern dessen Dissoziationskraft, so können wir einen neuen Zustand der Salzmolekeln herbeiführen, kurz, durch passende Wahl des dielektrischen Mediums können wir die vorliegenden Salzmolekeln die verschiedenartigen physikalischchemischen Zustände durchlaufen lassen, beginnend mit den hochpolymeren Molekeln, endend mit den einfachen lonen als letzten Bestandteilen, indem als Zwischenstufen sowohl polymere (komplexe) Ionen, als auch einfache (neutrale) Salzmolekeln auftreten müssen.

Augenscheinlich konnte eine experimentelle Prüfung dieser Annahrnen nur mit Hilfe eines typischen binären Elektrolyten vorgenommen werden, welcher in den zugänglichen lonisierungsmitteln, bis herab $z u$ den allerschwächsten, lös lich sein mubte. Die anorganischen binären Salze, etwa $\mathrm{KCl}, \mathrm{KJ}, \mathrm{NaJ}$, schieden wegen ihrer begrenzten Löslichkeit von vornherein aus. Die Wahl fiel daher auf die alkylsubstituierten Ammoniumsalze, und $z$ war die Jodide, und so wurden, auf Grund ihrer zunehmenden Löslich. keit in den schlechten lonisatoren bzw. Isolatoren, nacheinander im Laufe der Jahre verwendet: Tetraäthylammoniumjodid $\mathrm{N}\left(\mathrm{C}_{2} \mathrm{H}_{5}\right)_{4} \mathrm{~J}$, Tetrapropylammoniumjodid $\mathrm{N}\left(\mathrm{C}_{3} \mathrm{H}_{7}\right)_{4} \mathrm{~J}$, bzw. Tetra- 
(iso)amylammoniumjodid $\mathrm{N}\left(\mathrm{C}_{5} \mathrm{H}_{11}\right)_{4} \mathrm{~J}$. Sind nun diese Salze aber gleichartig und gleichwertig als Elektrolyte den typischen anorganischen binären Salzen, etwa dem Jodkalium KJ? Diese Prage wird von den hervorragendsten Vertretern der Elektrochemie ohne weiteres bejaht; so leitet Arrhenius ${ }^{2}$ ) als empirische Regel ab, ${ }_{n} \mathrm{daB}$
Salze desselben Typus bei gleicher molekularer Konzentration ungefähr gleich stark dissoziiert sind". Das Gesagte bezieht sich nun auf die wässerigen Lösungen. Für nichtwässerige Lösungen seien daher noch folgende Tatsachen als Beleg für die Gleichartigkeit, bzw. annähernde Gleichwertigkeit der fraglichen Salze angeführt.

\begin{tabular}{|c|c|c|}
\hline ypen & $\begin{array}{l}\text { Dissoziationsgrade } \\
\text { In Wasser }\left(t=25^{\circ}\right)\end{array}$ & $=\frac{\lambda_{\nu}}{\lambda_{\infty}}$ In Formamid $\left(t=25^{\circ}\right)$ \\
\hline $\mathrm{KCl} \cdot \cdot \cdot$ & $v=32 \ldots \alpha=\frac{126,0}{140,8}=0,89$ (Bredig) & $\nu=10, \alpha=\frac{23,27}{26,73}=0,87 \stackrel{\text { Jones }}{1915)}$. $^{\text {(J) }}$ \\
\hline $\mathrm{N}\left(\mathrm{C}_{2} \mathrm{H}_{5}\right)_{4} \mathrm{Cl}$ & $v=32 \ldots \alpha=\frac{88,2}{102,4}=0,86$ (Bredig) & 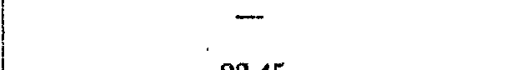 \\
\hline $\mathrm{KJ}\left(18^{\circ}\right)$ & $v=32 \ldots \alpha=\frac{118,8}{131,1}=0,91$ (Koh1rausch) & $v=10, \alpha=\frac{23,45}{26,28}=0,89$ (Jones) \\
\hline $\mathrm{N}\left(\mathrm{C}_{3} \mathrm{H}_{6}\right)_{6} \mathrm{~J}\left(\mathrm{t}=0^{0}\right)$ & $\nu=32 \ldots \alpha=\frac{47,74}{57,3}=0,84$ (Walden, 1899) & $\nu=10, \alpha=\frac{20,31}{24,28}=0,84(\mathrm{~J}$ \\
\hline
\end{tabular}

In Azetonitril $\left(t=25^{\circ}\right)$

$\mathrm{KJ}\left(\mathrm{t}=25^{\circ}\right) . .\left|\begin{array}{l}v=12 \ldots \alpha=\frac{102,4}{203}=0,50 \text { (Walden, 1905) } \\ \mathrm{N}\left(\mathrm{C}_{2} \mathrm{H}_{6}\right)_{4} \mathrm{~J}\left(\mathrm{t}=25^{9}\right)\end{array}\right| \begin{aligned} & v=10 \ldots \alpha=\frac{96,4}{200}=0,48 \text { (Walden, 1905) } \\ & v=14,6 \ldots \alpha=\frac{109,3}{200}=0,546 \text { (Walden, 1907) }\end{aligned}$

Das Verhalten dieser verschieden gebauten binären Salze kann wohl als ein gleichartiges bezeichnet werden, insofern sie alle zu den nahezu gleich stark dissoziierenden Elektrolyten gehören, sowohl den besten lonisatoren (Wasser und Formamid) als auch dem wesentlich schwächeren lonisierungsmittel (Azetonitril) gegenüber.

Die Gleichartigkeit des Verhaltens dieser und ähnlicher Salze ist auch ersichtlich, wenn man die neuerdings von mir $^{8}$ ) mitgeteilten $\mathrm{Be}-$ ziehungen zwischen den Dissoziationsgraden $\alpha$, bzw. den Dissoziationskonstanten Ko (nach Wilh. Ostwald) und den Dielektrizitätskonstanten $\varepsilon$ der lonisatoren in Betracht zieht.

Schlieblich mub noch verwiesen werden.auf die thleichen Beziehungen zwischen dem Grenzwert $\lambda_{\infty}$ und der Viskosität $\eta_{\infty}$ der Lösungsmittel, indem man aus den $\lambda_{\infty}$-Werten der verschiedenen Salze in den verschiedenen Medien mit $\eta_{\infty}$ das Produkt $\lambda_{\infty} . \eta_{\infty}=k 0 n s t$. jedes einzelne Salz erhält, so z. B. ${ }^{4}$ )

") Sv. Arthenius, Theorie der Chemie, II. Aufl. (1909), 215, 217; s. a. W. Nernst, Theoret. Chemie, VII. Aufl (1913), 546. (1920).

3. W W I den, Zeitschr. f. physik. Chem. 94, $263 \mathrm{ff}$.

4) Vgl. P. Wa I de n, Zeitschr. f. Elektrochem. 26, 68 (1920). $\lambda_{\infty} \cdot \eta_{\infty}=$ konst.

KJ $\quad \mathrm{N}\left(\mathrm{C}_{8} \mathrm{H}_{5}\right)_{4} \mathrm{~J} \quad \mathrm{~N}\left(\mathrm{C}_{3} \mathrm{H}_{7}\right)_{4} \mathrm{~J} \quad \mathrm{~N}\left(\mathrm{C}_{5} \mathrm{H}_{11}\right)_{4} \mathrm{~J}$ $\begin{array}{llll}0,650 & 0,700 & 0,624 & 0,557 .\end{array}$

Ohne Voreingenommenheit wird man daher neben den typischen anorganischen binären Salzefi (z.B. KJ, KCl) auch die tetraalkylierten Ammoniumsalze (Jodide) zu den gleichartig hochionisierten typischen starken Elektrolyten zählen milssen, wie das auch von Kraus und Bray (Journ. Amer. Chem. Soc. 35, 1319, 1913) geschieit. Mit Heranziehung dieser ,Normalelektrolyte ${ }^{\alpha}$ ist uun versucht worden, einen Querschnitt durch das ganze Gebiet der flüssigen organischen Lösungsmittel zu legen, um den Molekularzustand des gelösten Salzes zu beleuchten. Das Lösungsvermögen der einzalnen Solventien bedingte die fortschreitende Anreicherung der Alkylgruppen im Kation. Die Molekulargrößen wurden mit Hilfe der Gefrierbzw. Siedemethode bestimmt, wobei als Lösungsmittel nur diejenigen hierhergesetzt worden sind, welche zur lllustration der Rolle der che$m$ ischen Faktoren von etwaigem Werte sein dürften, dann aber zur Würdigung der physikalischen Faktoren, insbesondere der dissozilerenden Kraft der Medien, gemessen durch die Dielektrizitätskonstante $\varepsilon$ der letzteren, not- 
wendig sind. Von diesem Gesichtspunkte aus sind die Extreme in unserer Tabelle vertreten, d. h. das Formamid als eines der allerbesten, und der Tetrachlorkohlenstoff als eines der schlechtesten Ionisierungsmittel, welches bekanntlich zu den "Isolatoren" gezahlt wird. Die Ergebnisse der Messungen sind auf den Polymerie- oder Assoziationsgrad $x=\frac{M_{\text {get. }}}{M_{\text {theor. }}}$ umgerechnet worden, um ein anschauliches Bild von den Zustandsänderungen der gelösten Salzmolekeln zu lieférn. Die Verdŭ́nnung $V=4$ Liter (l $\mathrm{Mol}$ in 4 Litern) ist gewählt worden, um neben meinen Messungsergebnissen auch die wertvollen Daten von W. E. S. Turner zu verwenden, von dener ich, durch die Zeitumstande bedingt, erst unlängst an der Hand des Chem. Zentralbl. (1914 II, 1138 ) Kenntnis erhalten habe."

Verdünnung annahernd $\mathrm{V}=4$ Liter.

\begin{tabular}{|c|c|c|c|c|c|}
\hline \multirow[t]{2}{*}{ Lösungsmittel } & \multirow{2}{*}{$\begin{array}{l}\text { Dielektr.- } \\
\text { Konst. } \\
\varepsilon 20\end{array}$} & \multirow{2}{*}{$\underset{t^{0}}{\text { Versuchs- }}$} & \multicolumn{3}{|c|}{$\begin{array}{c}\text { Assoziations-(Polymerie-) Grad } x=\frac{M_{\text {get. }}}{M_{\text {theor. }}} \\
\text { für die Salze }\end{array}$} \\
\hline & & & $\mathrm{N}\left(\mathrm{C}_{8} \mathrm{H}_{6}\right)_{2} \mathrm{~J}$ & $\mathrm{~N}\left(\mathrm{C}_{3} \mathrm{H}_{7}\right)_{\mathrm{J}} \mathrm{J}$ & $\mathrm{N}\left(\mathrm{C}_{8} \mathrm{H}_{\mathbf{1 1}}\right)_{8} \mathrm{~J}$ \\
\hline $\begin{array}{l}\text { Formamid } \mathrm{HCONH}_{2} \text {. : } \\
\text { Nitromethan } \mathrm{CH}_{3} \mathrm{NO}_{2} \text {; : }\end{array}$ & ca. 94 & $+101^{\circ}$ & $\begin{array}{l}0,57 \\
0,66, v=4 \\
0,64, v=10\end{array}$ & - & - \\
\hline $\begin{array}{l}\text { Azetonitril } \mathrm{CH}_{8} \mathrm{CN} \\
\text { Nitrobenzol } \mathrm{C}_{6} \mathrm{H}_{5} \mathrm{NO}_{2}: \\
\text { Methylalkohol } \mathrm{CH}_{8} \mathrm{O}^{\circ}\end{array}$ & $\begin{array}{l}36,4 \\
35,5 \\
35,4\end{array}$ & $\begin{array}{c}81^{\circ} \\
5,8^{\circ} \\
66^{\circ}\end{array}$ & $\begin{array}{l}0,69, \quad=10 \\
0, \overline{80}, v=4\end{array}$ & $\begin{array}{l}0,60 \text { (Turner) } \\
0,81 \text { (Turner) }\end{array}$ & $0,76, \bar{z}=10$ \\
\hline Aethylalkohol $\mathrm{C}_{8} \mathrm{H}_{8} \mathrm{OH}$ & 25,4 & $78^{\circ}$ & $\begin{array}{c}0,22, v=10 \\
0,89, v=10 \\
0.99 v=4 \text { (Turner }\end{array}$ & 0.98 & - \\
\hline $\begin{array}{l}\text { Methylenchiorid } \mathrm{CH}_{2} \mathrm{Cl}_{4} \\
\text { Isoamiylalkohol } \mathrm{C}_{5} \mathrm{H}_{\mathrm{i1}} \mathrm{OH} \\
\text { Elsessig } \mathrm{CH}_{3} \mathrm{COOH} \text {. }\end{array}$ & $\begin{array}{c}8,3 \\
(\operatorname{cas} 5,7) \\
6,3-7,1\end{array}$ & $\begin{array}{c}42^{\circ} \\
132^{\circ} \\
16,5^{\circ}\end{array}$ & $\begin{array}{l}1,87 \text { (Turner) } \\
-(\text { schwerlost.) }\end{array}$ & $\begin{array}{l}>2 \\
2,12 \text { (Turnef) } \\
3,6 \text { (Walden) } \\
6\end{array}$ & $\overline{3,7}$ \\
\hline Chloroform $\mathrm{CHCl}_{\mathrm{s}}$ & 4,95 & $61^{\circ}$ & - (schwerlosl.) & 5 (Han & $>3$ \\
\hline $\begin{array}{l}\text { Benzol } \mathrm{C}_{6} \mathrm{H}_{6} \\
\text { Tetrachlorkohlenstoff } \mathrm{CCl}_{4}\end{array}$ & 3 & $\begin{array}{l}80^{\circ} \\
78^{\circ}\end{array}$ & $\begin{array}{l}- \text { (unlöslich) } \\
- \text { (unlosslich) }\end{array}$ & $\begin{array}{l}- \text { (unlöslich) } \\
- \text { (unlöslich) }\end{array}$ & $\infty$ \\
\hline
\end{tabular}

Literatur: P. Wald en, Zeitschr. f. physik. Chem. 55, 283 (1906); 94, 310 (1920); Zeitschr. f. Elektrochem. 26, $60 \mathrm{ff}$. (1920); Buil. Acad. Sc. St. Petersb. 1914, 1178; 1915, $233 \mathrm{ff} ., 509 \mathrm{ff}, 1499 \mathrm{ff}$ - - W. E. S. Turner, Joum. Chem. Soc. 105, 1751 (Chem. Centr. 1914 II, 1138); s. a. Journ. Chem. Soc. 99, 891 ( $\left.\mathrm{CHCl}_{8}\right)$. Hantzs ch, Ber. d. Deutsch. chem. Ges. 44, 1779 (1911), s. a. 42, 76 ff.

Das allgemeine Ergebnis läkt sich dahin zusammenfassen, daß zwischen dem Assoziationsgrade $x$ eines gegebenen Salzes und der Dielektrizitätskonstante $\varepsilon$ der Lösungsmittel ein sichtbarer Zusammenhang besteht, und zwar nimmt der Assoziationsgrad $x$ mit fallender Dielektrizitätskonstante $z u$, erreicht also seine höchsten Werte in den Medien mit der geringsten Dielektrizitätskonstante. Das Solvens wirkt daher auf die Salzmolekeln gleichsam wie eine Molekular-(Kugel-) Mühle, indem es die polymeren Salzmolekeln bei gegebener Verdünnung, je nach seiner Dielektrizitätskonstante $\varepsilon$, in verschieden fein verteiltem Zustande, mit wechseinder "molekularer KorngröBe" liefern kann.

Unser besonderes Interesse erwecken nach dem eingangs Dargelegten die Polymeriegrade in den beiden "Isolatoren" Benzol und Tetrachlorkohlenstoff. Hier haben wir für die Asso- ziation den Wert $x=\infty$ eingezeichnet. Das gelöste Salz, das in den anderen Medien sich normal verhielt, würde in diesen Isolatoren in einem Zustande sein, welcher ähnlich den Kollo iden wäre, für welche die Siedetemperaturerhöhungen ebenfalls praktisch gleich Null (also $\mathbf{x}=\infty$ ) betragen.

Um zu veranschaulichen, wie die Temperatur des Siedens in Abhängigkeit von der Konzentration sich in den Lösungen von Benzol und Chlorkohlenstoff änderte, seien einige Versuchsreihen hierhergesetzt.

\section{Benzol als Lösungsmittel.}

Das Benzol (Kahlbaum) wurde andauernd über metall. Natrium gekocht und über frisch. Na abdestilliert. Als Wert für die Siedekonstante wurde $K=26,7$ gewählt. Dichte beim Siedep. $\mathrm{d}_{80 / 4}=0,811$ (Schiff). 
Salz: Tetraamylammoniumjodid $N\left(\mathrm{C}_{5} \mathrm{H}_{11}\right)_{4}$ d. Mol.-Gew. $M=425$.

1. Versuchsreibe.

\begin{tabular}{|c|c|c|}
\hline $\begin{array}{c}\mathrm{p}-\mathrm{g} \text { Substi. } \\
\text { auf } 100 \mathrm{~g} \text { Solvens } \\
0 .\end{array}$ & $\begin{array}{l}\text { beobachtete } \\
\text { Siedetemp. } \\
. \quad 4,151^{\circ} \mathrm{C} \\
\end{array}$ & $\Delta t$ \\
\hline $2,02(V=26$ Lit $)$ & $4,124>-0,027^{\circ}$ & $-0,027^{\circ}$ \\
\hline 4,01 . & $4,108>-0,016$ & $-0,043$ \\
\hline 7,08 & - $4,096>-0,012$ & $-0,055$ \\
\hline 10,70 & $4,114>+0,018$ & $-0,037$ \\
\hline $13,92(V=3,77)$ & $-0,002$ & $-0,039$ \\
\hline
\end{tabular}

Die Lősung wà gelblich gefärbt.

il. Versuchsreihe, mit einer andern Probe des Salzes.

\begin{tabular}{|c|c|}
\hline $0 \ldots .$. & $\therefore 4,788^{\circ}$ \\
\hline $\begin{array}{l}1,33(V=40) \\
3,02 \quad \therefore \\
5,14: \therefore \\
7,14: 0 \\
9,30(V=5,6)\end{array}$ & $\left.\begin{array}{r}\because 4,8111^{\circ} \\
\therefore 4,812 \\
\therefore 4,809 \\
\therefore 4,813 \\
\quad 4,813\end{array}\right\}$ \\
\hline
\end{tabular}

In beiden Versuchsreihen ist der Siedepunkt der Lösungen - nach der ersten Stoffzugabe innerhalb der Pehlergrenzen konstant, also bis zu 9 bzw. 14 Proz. hinauf von der zugegebenen Salzmenge unabhängig. Im ersten Fall trat eine geringe Verminderung des Siedepunkts auf $\left(\triangle t=-0,040^{\circ}\right)$, mit der zweiten Salzprobe eine kleine Temperaturerhöhung $\left(\Delta t=+0,024^{\circ}\right)$. Das Sieden zeigte keine Anomalien, die besonders auffällig gewesen wären. Aus den abgekühtten Lösungen kristallisierte das Salz heraus, und zwar als Salzberzolat, also mit Kristallbenzol, das leicht wegdunstete.

Verfolgt man dasselbe Konzentrationsgebiet ( $V=4-40$ Lit.) mit Hilfe der Messungen des elektrischen Leitvermögens, so ergibt sich das folgende Bild $(\lambda u=$ Molekularleitvermögen $)$.

In Benzol. Salz: $N\left(C_{5} H_{11}\right)_{4}$ J. $t=25^{\circ}$.

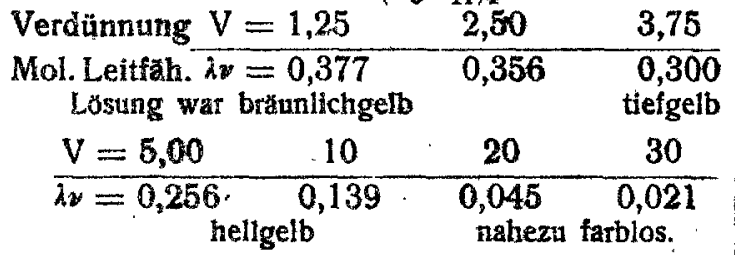

Konzentrierte Lösungen: $t=25^{\circ}$.

\begin{tabular}{lllll}
$V=0,75$ & 1,125 & 1,50 & 2,25 & 3,00 \\
\hline$\lambda v=0,243$ & 0,372 & $\begin{array}{c}0,391 \\
\text { Maxim. }\end{array}$ & 0,363 & $0,323$.
\end{tabular}

Die benzolische Losung des Salzes ist also ein relativ guter Stromleiter; um dieses besser zu veranschaulichen, setze ich folgende Vergleichswerte hierher:

Kobaltchlorid $\frac{\mathrm{CoCl}_{2}}{2}$ in Amylalkohok, $\mathrm{t}=20^{\circ}$, $\mathrm{V}=3,6, \lambda \nu=0,042$ (W. Hardt);

Tripropyla m in chlorid $\mathrm{N}\left(\mathrm{C}_{3} \mathrm{H}_{7}\right)_{3} \mathrm{HCl}$ in Azeton, $\mathrm{t}=25^{\circ}, \mathrm{V}=4,0, \lambda v=1,542$ (Walden);

obiges Salz $\mathrm{N}\left(\mathrm{C}_{5} \mathrm{H}_{11}\right)_{4} \mathrm{~J}$ in Benzol, $\mathrm{t}=25^{\circ}$, $V=3,75, \lambda v=0,300$;

Silbernitrat $\mathrm{AgNO}_{3}$ in Anilin, $t=25^{\circ}, \mathrm{V}=4$, $\lambda \nu=0,87$ (Pearce).

Die Anomalie in der Kurve $\lambda \nu-V$, d. h. das Ansteigen zu einem Maximum in großen Konzentrationsgebieten (gewöhnlich bei $V=1-2$ ), Abnahme von $\lambda \nu$ mit zunehmender Verdünnung und Passieren eines Minimums, ist eine für schlechte Ionisatoren normale Erscheinung, die den Forschern auf diesem Gebiete.wohl bekannt ist und daher im Falle der benzolischen Lösungen nichts Außergewöhnliches darstellt. Kurz gesagt, auf Grund der Leitfähigkeitsmessungen der benzolischen Lösungen des Tetraamylammoniumjodids sind keinerlei Anomalien im ebullioskopischen Verhalten solcher Lösungen vorauszusehen. Daß der besondere Gang der $\lambda_{\nu}$-Werte mit der Verdünnung.V. sich : ebenso findet bei demselben Salz $N\left(\mathrm{C}_{5} \mathrm{H}_{11}\right)_{4} \mathrm{~J}$ in $\mathrm{Ch}$ lo r of orm lösungen, trotzdem. nunmehr keine aufergewöhnliche Assoziation vorliegt, da $x>3$ gefunden wurde, beweisen folgende Zahlen :

In Chloroform. Salz: $\mathrm{N}\left(\mathrm{C}_{5} \mathrm{H}_{11}\right)_{4}$ J. $\mathrm{t}=25^{\circ}$.

\begin{tabular}{lllll}
$\mathrm{V}=5,0^{\circ}$ & 7,5 & 10 & 20 & 50 \\
\hline$\lambda \nu=3,185$ & 2,81 & 2,45 & 1,57 & $0,74$.
\end{tabular}

\section{Tetrachlorkohlenstoff als Lösungsmittel.}

Kahlbau'm'sches Prăparat wurde über frisch geglühter Pottasche getrocknet (häufiges Schütteln und längeres Stzhenlassen), dann über geglühter Pottasche destilliert; die bei $77,0^{\circ} \mathrm{C}$ übergegangene Fraktion (bei $770 \mathrm{~mm}$ ) wurde dann $\mathbf{z u}$ den Molekulargewichtsbestimmungen im Beckmann'schen Apparat verwendet. Unter Einsetzung der theoretisch aus der Verdampfungswärme berechneten Konstante $K=52,8$ wurden Kontrollversuche mit Benzoesäuremethylund -äthylester, sowie p-Toluidin ausgeführt, welche die normalen Molekulargrößen innerhalb +2 Proz. lieferten. 
Salz: Tetr a my la m moni umjodid $\mathrm{N}\left(\mathrm{C}_{5} \mathrm{H}_{11}\right)_{4} \mathrm{~J}$. Mol.-Gew. $M=425$.

Versuchsreihe. Das Salz war aus Benzol umkristallisiert, besaß den Schmelzpunkt $144^{\circ}$ und stellte farblose Blättchen dar.

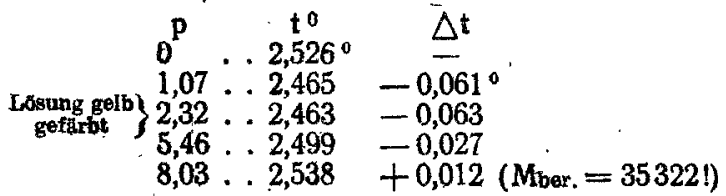

Wollten wir mit der bei der letzten Konzeniration erzielten positiven Temperaturänderung $\Delta \mathrm{t}=+0,012$ das Molekulargewicht berechnen, so würden wir zu $M=\frac{52,8 \cdot 8,03}{0,012} \sim 35300$ gelangen, also eine Komplexheit der Salzmolekel $x=\frac{35300}{425} \sim 80$, bzw. $[M]_{80}$ erhalten.

In demselben Solvens wurde nun parallel ein anderes Salz gemessen, um zu sehen, ob die Konstitution des Elektrolyten die beobachteten Anomalien im vorigen Fall bedingt.

$$
\begin{aligned}
& \text { Salz: Triamylaminpikrat } \\
& \mathrm{N}\left(\mathrm{C}_{5} \mathrm{H}_{11}\right)_{3}: \mathrm{C}_{6} \mathrm{H}_{2}\left(\mathrm{NO}_{2}\right)_{3} \mathrm{OH} \text {. } \\
& \text { Mol.-Gew. } \mathrm{M}=456 .
\end{aligned}
$$

$$
\begin{aligned}
& \text { Mget. } \\
& 2,52 \text {. . 1211 } \text { Assoziationsgrad }
\end{aligned}
$$

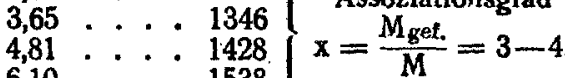

$$
\begin{aligned}
& 6,10 \ldots 1538
\end{aligned}
$$

Das Pikrat erweist sich als normal, d. h. es gibt mebbare, mit der Konzentration zunehmende Siedepunktserhöhungen und führt zu Molekulargrößen, welche eine drei-bis vierfache Assoziation der Salzmolekeln bekunden. In schlechten Ionisatoren mit geringer Dielektrizitätskonstante (hier ist die Diel.- Konst. $=2,18$ ) ist eine solche Assoziation die gewöhnliche $\mathrm{Er}$ scheinung.

Das anomale Verhalten des Tetraamylammoniumjodids $\mathrm{N}\left(\mathrm{C}_{5} \mathrm{H}_{11}\right)_{4} \mathrm{~J}$ legt nun noch die
Frage nahe, ob es in Chlorkohlenstofflösungen überhaupt ein Elektrolyt ist, und wie seine Leitfähigkeitskurve mit der Verdünnung verläuft? Nachstehende Zahlen geben die Antwort.

$$
\text { Salz: Tetraamylammonium jodid }
$$

$$
\mathrm{N}\left(\mathrm{C}_{5} \mathrm{H}_{11}\right)_{4} \mathrm{~J} . \cdot \mathrm{t}=25^{\circ} \text {. }
$$

$$
\begin{aligned}
& \begin{array}{ccc}
\text { Verdünnung } V=2,5 & 5 \text { I } & 5_{\mathrm{II}} \\
\text { Mol. Leitf. } \lambda v=0,0340 & 0,0245 & 0,0244
\end{array} \\
& \begin{array}{lcc}
V=7,5 & 10 & 20 \text { Lit. } \\
\hline \lambda \nu=0,0216 & 0,0199 & 0,0140 .
\end{array}
\end{aligned}
$$

Threr $G r o ̈ B e$ nach erinnern ans die $\lambda \nu$. Werte an die Leitfähigkeit von Kobaltchlorid $\frac{\mathrm{CoCl}_{2}}{2}$ in Amylalkohol (s. vorhin beim Benzol), der Gang der Leitfähigkeit iv mit der Verdünnung ist ähnlich dem Verhalten in Benzollösungen bzw. in Chloroform: Ob die elektrische Leitfähigkeit in den Benzol- und Chlorkohlenstofflösungen durch eine äuBerst geringe lonenspaltung bedingt wird, ist fraglich, eher könnte man an elektrisch geladene Molekülkomplexe denken.

Ueberblicken wir abschliefend das Dargelegte, so kommen wir zu dem Schluk, daB die hochpolymeren binären Elektrolyte, bzw. typischen Salze beliebig alle Zustände der Molekularkomplexität durchlaufen können, indem man sie aus dem festen kristallinischen Zustand durch geeignete Lösungsmittel in den gelösten Zustand überführt. Solventien mit den kleinsten Dielektrizitätskonstanten $(\varepsilon>2)$ üben auf die Molekularkomplexität solcher Salze eine Schutzwirkụng aus, indem sie den Polymeriegrad der Salzmolekeln auf einer Höhe erhalten, welche den Kolloiden gleichkommt. Vom Standpunkt der Kolloidchemie kann man dann mit Wo. Ostwald sagen, dab dieselbe nicht so sehr, von kolloiden $S$ toffarte $n$, als vielmehr von kol- loiden St off $z$ uständen zu handeln hat. Rostock, 4. Juni 1920.

Chemisches Institut der Universital.

\section{Der Böschungswinkel - eine neue physikalische Konstante.}

\section{Von A. Langhans (Siegburg).}

(Eingezangen 6. Mai 1920.)

Wie der Name Bö́schungswinkel andeutet, handelt es sich um die Bezeichnung und Bestimmung eines Wertes, der für Böschungen, d. h. für natürliche oder künstliche Aufschüttungen von Erd-oder Steinmassen charakteristisch ist. Es ist der Winkel, den die Seitenlinie dieser
Aufschüttung mit der Horizontalen, in den an* gedeuteten Fällen also mit dem Erdboden bildet; er ist vor allen Dingen bestimmend für die Belastung von oben oder die seitliche Beanspruchung, die die Böschung vertragen kann, ohne ihren Aufbau und ihren Halt zu verlieren, 